\title{
Dynamical Model about Rumor Spreading with Medium
}

\author{
Xiaxia Zhao ${ }^{1,2}$ and Jianzhong Wang ${ }^{1,2}$ \\ ${ }^{1}$ National Key Laboratory for Electronic Measurement Technology, North University of China, Taiyuan, Shanxi 030051, China \\ ${ }^{2}$ Laboratory of Instrumentation Science and Dynamic Measurement, Ministry of Education, North University of China, \\ Taiyuan, Shanxi 030051, China
}

Correspondence should be addressed to Xiaxia Zhao; zxxzbdx@sina.cn

Received 13 December 2012; Accepted 22 January 2013

Academic Editor: Yanbin Sang

Copyright (c) $2013 \mathrm{X}$. Zhao and J. Wang. This is an open access article distributed under the Creative Commons Attribution License, which permits unrestricted use, distribution, and reproduction in any medium, provided the original work is properly cited.

\begin{abstract}
Rumor is a kind of social remark, that is untrue, and not be confirmed, and spreads on a large scale in a short time. Usually, it can induce a cloud of pressure, anxiety, and panic. Traditionally, it is propagated by word of mouth. Nowadays, with the emergence of the internet, rumors can be spread by instant messengers, emails, or publishing. With this new pattern of spreading, an ISRW dynamical model considering the medium as a subclass is established. Beside the dynamical analysis of the model, we mainly explore the mechanism of spreading of individuals-to-individuals and medium-to-individual. By numerical simulation, we find that if we want to control the rumor spreading, it will not only need to control the rate of change of the spreader subclass, but also need to control the change of the information about rumor in medium which has larger influence. Moreover, to control the effusion of rumor is more important than deleting existing information about rumor. On the one hand, government should enhance the management of internet. On the other hand, relevant legal institutions for punishing the rumor creator and spreader on internet who can be tracked should be established. Using this way, involved authorities can propose efficient measures to control the rumor spreading to keep the stabilization of society and development of economy.
\end{abstract}

\section{Introduction}

Rumor is a kind of social phenomenon that a remark spreads on a large scale in a short time through chains of communication and runs through the whole evolutionary history of mankind [1]. Narrowly speaking, rumor is defined as untrue information, but has not been confirmed. Usually, it is dispersed by some people in order to achieve the specific purpose: slandering others, manufacturing momentum, diverting attention, causing panic, and so on [2-4]. Most rumors induce panic psychology or economic loss in the accompanying unexpected events. Emergencies cause serious negative impacts on people's life in several ways: not only the event itself might lead to financial loss or personal injuries, but also the rumor might lead to panic feelings and irrational behavior [5]. For example, the nuclear leakage in Japan caused an alt-buying frenzy in China. With the rumors spreading, this frenzy swept and caused social panic in just a few days and the preternatural rising of the salt price, which has a negative effect on society and economy [6]. Recently, there appeared a hearsay that beef in Suzhou city was infected bacillus anthracis; but after a few days, the rumor cannot stand and is confirmed.

The spreading of rumor is in many ways similar to the spreading of epidemic infection, by the spreader or infectious to notify or infect the susceptible. After notified or infected, that the spreader can become the stifler is similar as the infectious can recover after a few time. Thus many epidemic models have been used to describe the spread of information in the form of rumors [6-11]. A classical model for the spread of rumor was introduced by Daley and Kendall [12] who first proposed the basic DK model of rumor spreading. Maki and Thompson [13] focused on the analysis of the rumor spreading model, which was based on mathematical theory and developed the MK model. The DK and MK models have been used extensively for quantitative studies of rumor spreading [14-16]. The literatures $[5,17,18]$ give a detailed introduction for the models about rumor spreading.

Traditionally, rumors are propagated by word of mouth. In this propagation mode, the spreading speed is slower and 
TABLE 1: Description of parameters in the model (5).

\begin{tabular}{lccl}
\hline Parameters & Value & Unit & Comments \\
\hline$\beta$ & 10 & Day $^{-1}$ & $S(t)$-to- $I(t)$ transmission rate. \\
$\alpha$ & 0.00001 & Day $^{-1}$ & $W(t)$-to- $I(t)$ transmission rate. \\
$\theta$ & 0.5 & None & The rate of being $S(t)$ after transmission. \\
$m$ & 0.1 & Day $^{-1}$ & The automatical transformation rate of $S(t)$ into $R(t)$. \\
$k$ & 2 & Day $^{-1}$ & The submerged rate of message. \\
$\lambda$ & 5 & Day $^{-1}$ & The discharge quantity of message into medium. \\
$\xi_{1}$ & 15 & Day $^{-1}$ & $S(t)$-to- $S(t)$ transmission rate. \\
$\xi_{2}$ & 15 & Day $^{-1}$ & $R(t)$-to- $S(t)$ transmission rate. \\
$I(0)$ & 5000000 & Individual $^{-1}$ & The initial number of the susceptible individual. \\
$S(0)$ & 10 & Individual $^{-1}$ & The initial number of the spreader. \\
$R(0)$ & 0 & Individual & The initial number of the stifler. \\
$W(0)$ & 0 & The initial quantity of message.
\end{tabular}

can produce a small effect on society stability. Nowadays, with the emergence of the internet, rumors can spread rapidly by instant messengers, emails, or publishing bloggings that provide faster velocity of transmission and result in widespread of information [6]. Under this case, it is more difficult to control the rumor spreading. The paper about infective vector can be seen in $[19,20]$. With this new pattern of rumor spreading, we establish an ISRW dynamical model including spreading between individuals and medium-toindividuals to describe more accurately the actual pattern of transmission, which has not been studied in previous papers. Then the mechanism under spreading between individuals and by medium can be investigated by resorting to the model. Furthermore, we also give the main influence factors of transmission to government that can propose efficient measures to keep the stabilization of society and development of economy.

\section{A Dynamical Model for Rumor Spreading}

The populations at any time $t$ we consider are denoted by $N(t)$, which is classified into three subclasses: the susceptible or ignorant, spreader, and stiflers denoted by $I(t), S(t)$, and $R(t)$, respectively. Besides the rumor spreading from $I(t)$ to $S(t)$, the susceptible can be notified by medium, such as website news, microblog, Baidu Post Bar, community sites, Renren, and mobile phone. The quantity of rumor in medium is denoted by $W(t)$. The mathematical model to study the rate of change of the populations is given by system (5). Our assumptions on the dynamical transmission of rumor among humans are demonstrated in the following flowchart (Figure 1).

When a susceptible contacts a spreader or sees rumor in medium, the susceptible can get to know about the rumor. Thus, the change over time in the size of the susceptible population $I(t)$ only consists of the output, infected by the rumor $-\beta(I(t) S(t) / N)-\alpha I(t) W(t)$. For the incidence rate under direct contact between individuals, we adopt the standard incidence rate. According to the indirect infection between the susceptible individual and the rumor in medium, the probability to be infected for a susceptible individual is

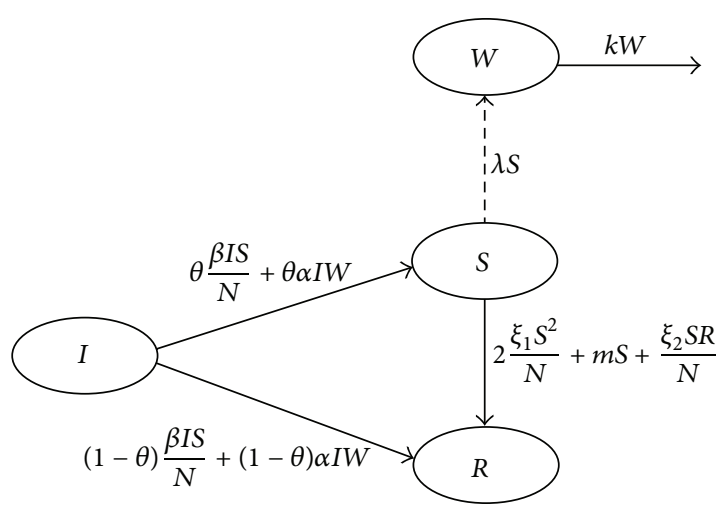

FIGURE 1: Transfer diagram on the dynamical transmission of rumor among humans.

proportional to the quantity of rumor in medium. So, the indirect transmission rate is $\alpha I(t) W(t)$. With regard to $W(t)$, it is very difficult to determine the quantity in medium. In addition, for the susceptible, after notified rumor, they do not completely become the rumor, but can doubt its credibility and become a stifler. Thus, the susceptible notified rumor enters the spreader at a constant rate $\theta \in[0,1]$ per unit time. Consequently, the change over time in the size of the susceptible dairy cattle population $S(t)$ is as follows:

$$
\frac{d I(t)}{d t}=-\beta \frac{I(t) S(t)}{N}-\alpha I(t) W(t) .
$$

The interpretation for the change over time in the size of the spreader population $S(t)$ is as follows. Once infected, the individuals will enter the spreader compartment at a constant rate $\theta \in[0,1]$ per unit time. However, when two spreaders contact, both of them transmit the rumor, and the spreader can become bored and loses interest in the rumor, and consequently both become stiflers. In this case, the term $\xi_{1} S^{2}(t) / N$ needs 2 times. During a unite time, the number of the spreader $m S(t)$ also can realize truth with the development of the matter. When a spreader contacts a stifler 

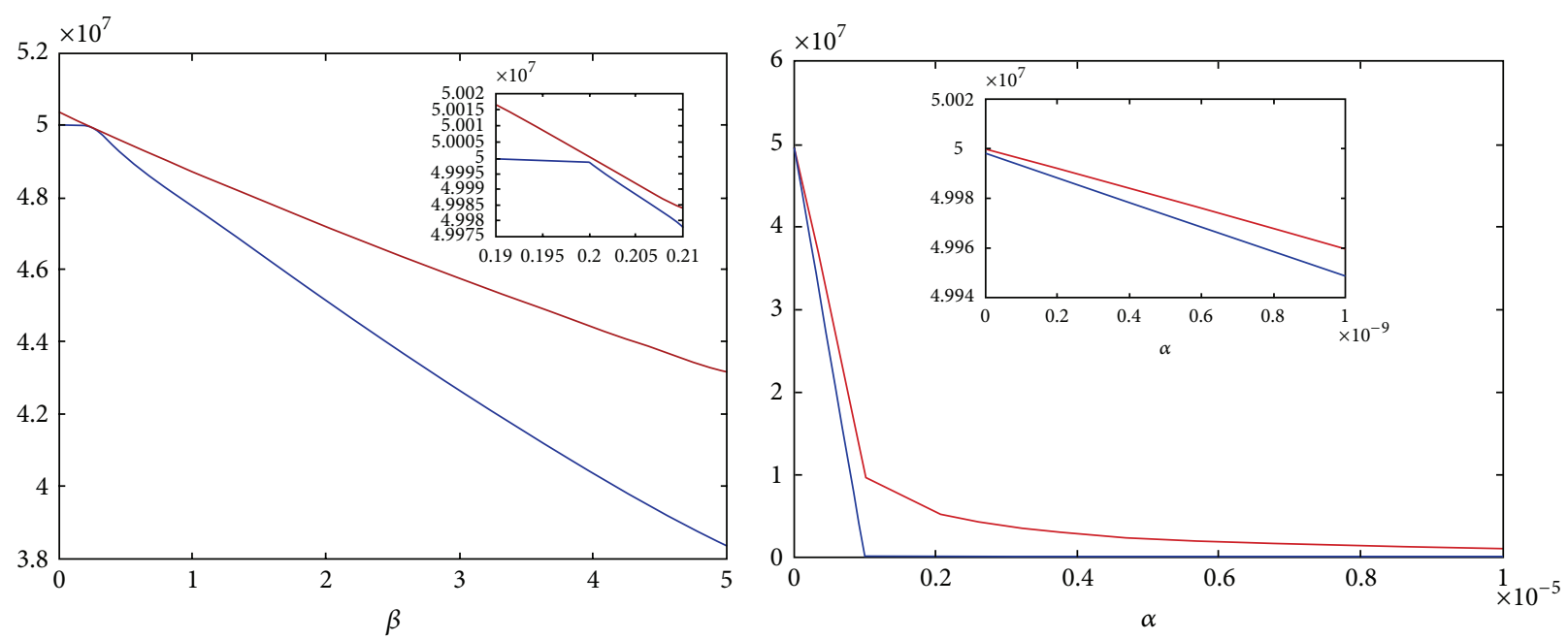

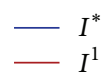

(a)
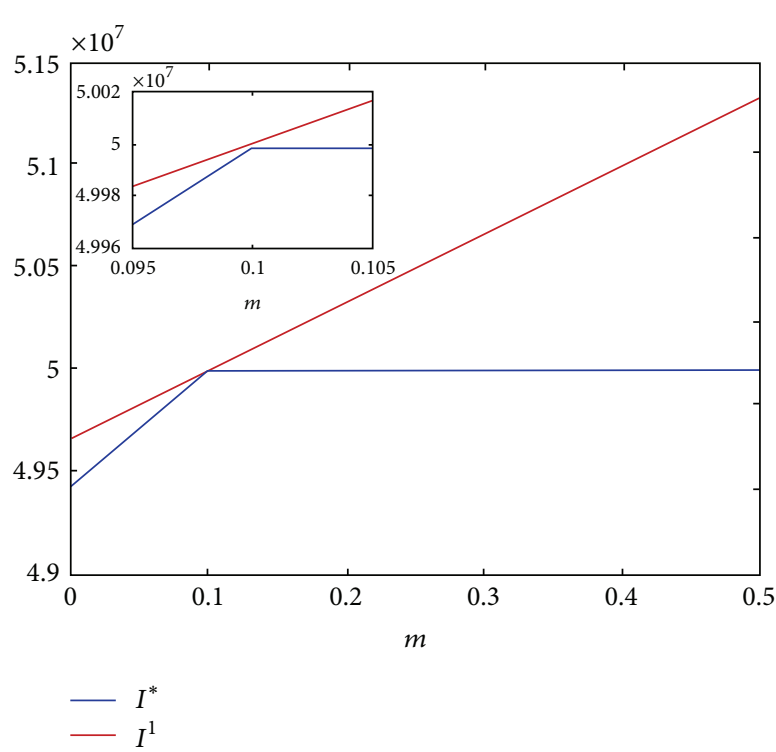

$-I^{*}$
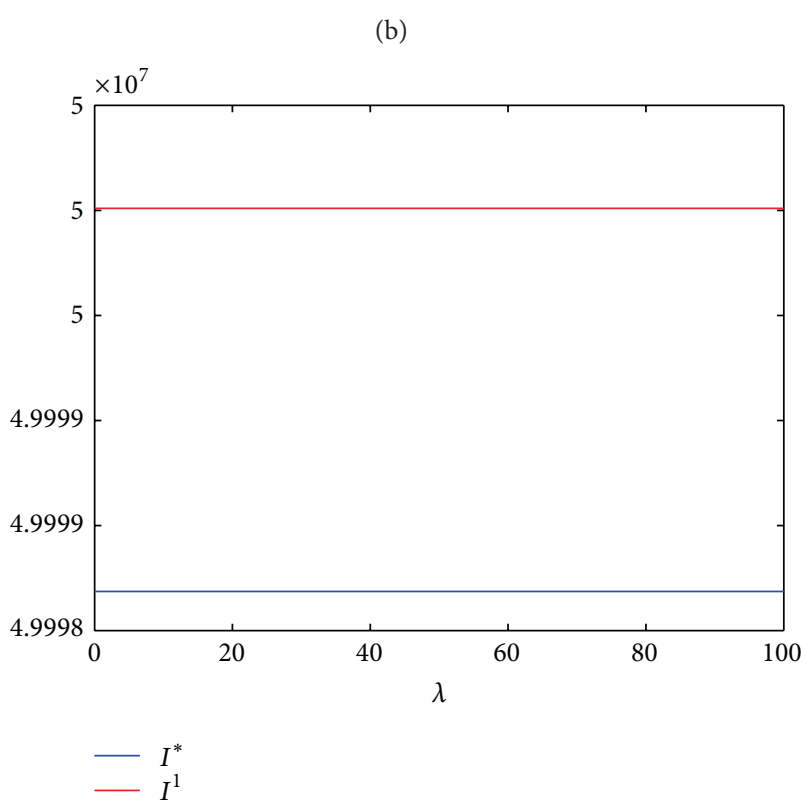

(d)

FIGURE 2: The comparison of $I^{*}$ with $I^{1}$ : (a) in terms of $\beta$, where $\alpha=0$, (b) in terms of $\alpha$, where $\beta=0.2$, (c) in terms of $m$, where $\beta=0.2$ and $\alpha=0$, and (d) in terms of $\lambda$, where $\beta=0.2$ and $\alpha=0$. The other parameter values and the initial values can be seen in Table 1 , unless we say otherwise.

and transmits the rumor, the stifler tries to remove it, and the spreader becomes the stifler at a rate of $\xi_{2} S(t) R(t) / N$ :

$$
\begin{aligned}
\frac{d S(t)}{d t}= & \theta \beta \frac{I(t) S(t)}{N}+\theta \alpha I(t) W(t)-2 \frac{\xi_{1} S^{2}(t)}{N} \\
& -m S(t)-\frac{\xi_{2} S(t) R(t)}{N} .
\end{aligned}
$$

On the one hand, there are $(1-\theta) \beta(I(t) S(t) / N)+(1-$ $\theta) \alpha I(t) W(t)$ from the susceptible department to the stifler department. On the other hand, there are $\xi_{1} S^{2}(t) / N, m S(t)$, and $\xi_{2} S(t) R(t) / N$ from the spreader department to the stifler class. Thus, the change over time in the size of the stifler population $R(t)$ is as follows:

$$
\begin{aligned}
\frac{d R(t)}{d t}= & (1-\theta) \beta \frac{I(t) S(t)}{N}+(1-\theta) \alpha I(t) W(t) \\
& +2 \frac{\xi_{1} S^{2}(t)}{N}+m S(t)+\frac{\xi_{2} S(t) R(t)}{N} .
\end{aligned}
$$

For the rumor in medium, they are discharged by the spreader, and the quantity discharged per unite time is assumed as $\lambda S(t)$. However, they have the submerged rate of message $k W(t)$. The quantity of rumor in medium is close to the follows of a person in network, so its unit also can be 


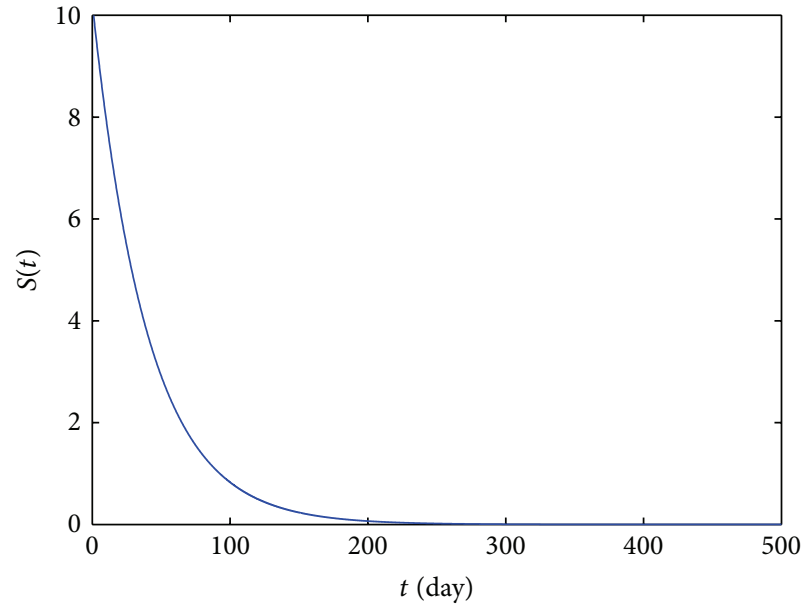

(a)

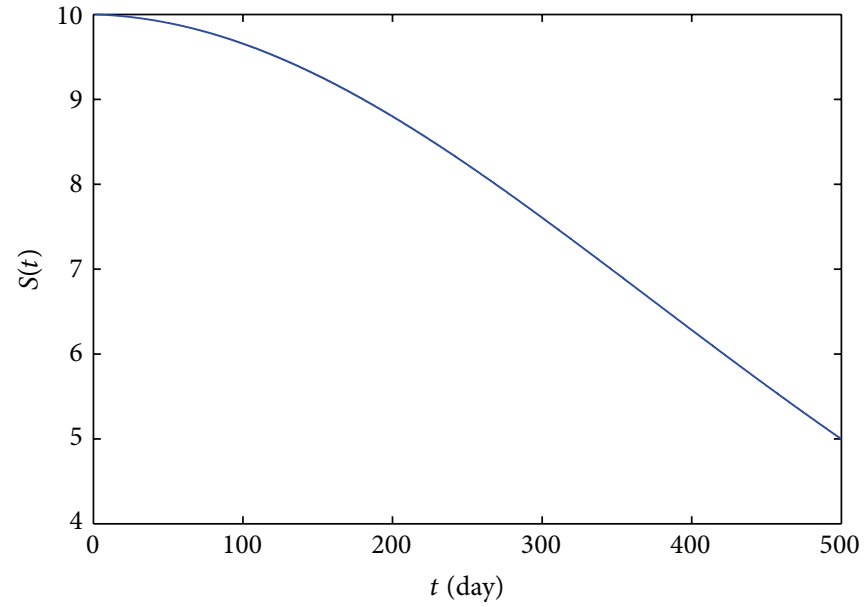

(b)

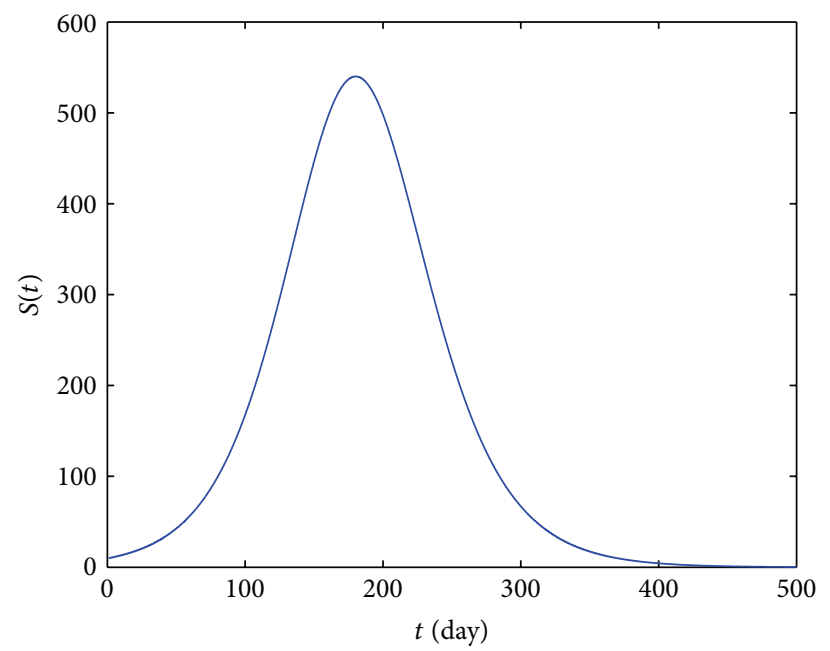

(c)

FIGURE 3: The number of the spreaders in terms of time under different condition, (a) $d I / d t=-0.2506$, where $\beta=0.15$, (b) $d I / d t \approx 0$, where $\beta=0.200005$, and (c) $d I / d t=0.2994$, where $\beta=0.26$. The other parameter values and the initial values can be seen in Table 1 .

individual. Thus, the change over time in the size of the $W(t)$ is as follows:

$$
\frac{d W(t)}{d t}=\lambda S(t)-k W(t)
$$

Combining the above equations, the model we study in this paper to govern the temporal evolution of rumor during human population is presented in (5), whose parameters are described in Table 1. In the next section, we will discuss the dynamical behavior of model

$$
\begin{aligned}
\frac{d I(t)}{d t}= & -\beta \frac{I(t) S(t)}{N}-\alpha I(t) W(t) \\
\frac{d S(t)}{d t}= & \theta \beta \frac{I(t) S(t)}{N}+\theta \alpha I(t) W(t)-2 \frac{\xi_{1} S^{2}(t)}{N} \\
& -m S(t)-\frac{\xi_{2} S(t) R(t)}{N}
\end{aligned}
$$

$$
\begin{aligned}
\frac{d R(t)}{d t}= & (1-\theta) \beta \frac{I(t) S(t)}{N}+(1-\theta) \alpha I(t) W(t) \\
& +2 \frac{\xi_{1} S^{2}(t)}{N}+m S(t)+\frac{\xi_{2} S(t) R(t)}{N}
\end{aligned}
$$

$$
\frac{d W(t)}{d t}=\lambda S(t)-k W(t)
$$

\section{Dynamical Behavior of Model (5)}

Because $d N(t) / d t=0, N(t)$ is independent of time and is a constant, denoted by $N^{*}$. We can obtain the equilibrium of the system (5) $E_{*}=\left(I^{*}, 0, R^{*}, 0\right)$, where $I^{*}+R^{*}=N^{*}$; that is, the rumor must disappear with time, and all $I^{*}$ and $R^{*}$, that satisfy $I^{*}+R^{*}=N^{*}$, represent stable situations. Next, we have a look at the stability of the equilibrium $E_{*}=\left(I^{*}, 0, R^{*}, 0\right)$. 


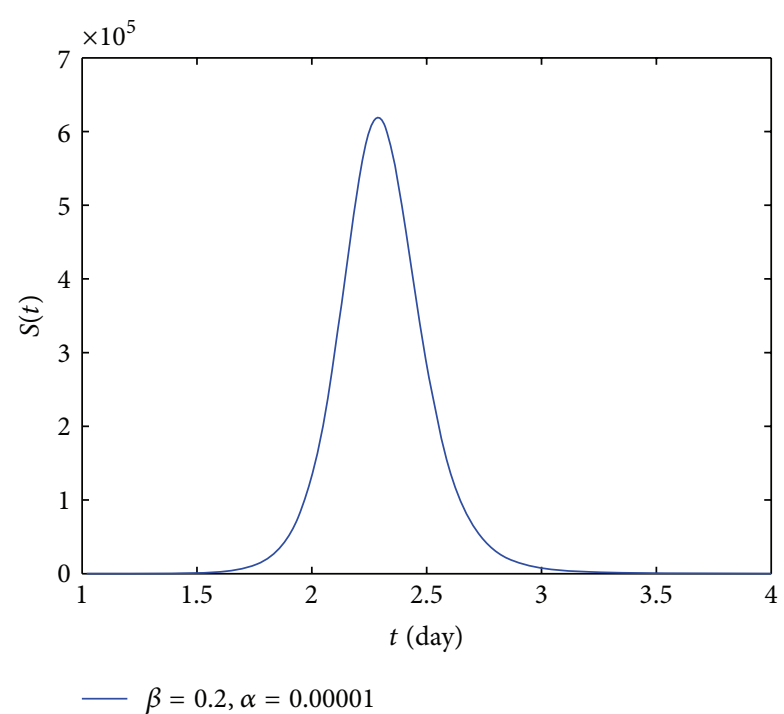

FIgURE 4: The number of each subclass in terms of time under different conditions, where $I(0)=5000000, S(0)=10, R(0)=$ $0, W(0)=0, \beta=0.2, \alpha=0.00001, d I / d t=-0.000602$, and $d W / d t=50$.

Theorem 1. The equilibrium $E_{*}$ is globally asymptotically stable.

Proof. In order to verify the stability of these points, the linear part of the vector field around them is given by the Jacobian at the equilibrium $E_{*}=\left(I^{*}, 0, V^{*}, 0\right)$ as follows:

$$
\left.J\right|_{E_{*}}=\left(\begin{array}{cccc}
0 & -\frac{\beta I^{*}}{N^{*}} & 0 & -\alpha I^{*} \\
0 & \frac{\theta \beta I^{*}}{N^{*}}-m-\frac{\xi_{2} R^{*}}{N^{*}} & 0 & \theta \alpha I^{*} \\
0 & \frac{(1-\theta) \beta I^{*}}{N^{*}}+m+\frac{\xi_{2} R^{*}}{N^{*}} & 0 & (1-\theta) \alpha I^{*} \\
0 & \lambda & 0 & -k
\end{array}\right) .
$$

With regard to the above matrix, we know that one zero eigenvalue corresponds to the fact that the order of the dynamical system is two. The other zero eigenvalue is related to the stable center manifold that is the straight line $I^{*}+V^{*}=$ $N^{*}$ on the $\left(I^{*}, V^{*}\right)$ plane. Thus, the stability of the equilibrium depends on the signal of the remainder eigenvalues. The corresponding characteristic polynomial can be written as

$$
\begin{aligned}
x^{2}+ & \left(k-\frac{\theta \beta I^{*}}{N^{*}}+m+\frac{\xi_{2} R^{*}}{N^{*}}\right) x \\
& -k\left(\frac{\theta \beta I^{*}}{N^{*}}-m-\frac{\xi_{2} R^{*}}{N^{*}}\right)-\lambda \theta \alpha I^{*}=0 .
\end{aligned}
$$

Using $I^{*}+R^{*}=N^{*}$ and the Hurwitz criterion, we can get when $0<I^{*}<I^{1}=N^{*} k\left(m+\xi_{2}\right) /\left(\lambda \theta \alpha N^{*}+\xi_{2} k+\theta \beta k\right)$, $E_{*}$ is locally asymptotically stable. In fact, applying the twodimensional figures, we can know that when $I^{*}$ is always less than $I^{1}$. Here, we give figures about $\beta, \alpha, m$, and $\lambda$, seen in Figure 2 .

From Figure 2, we can see when $\beta \leq 0.2$, the red curve, representing $I^{1}$, is a constant 5000000 , and always over the blue line, $I^{*}$. At $\beta=0.2$, the two curves are very closer, but the gap can be observed by observing the microdiagram round $\beta=0.2$. When $\beta>0.2$, the gap between them becomes larger. At $\beta=0.2$, we have a look at the change of $I^{*}$ and $I^{1}$ in terms of the parameter $\alpha$. It can be observed that, at $\alpha=0$, two curves are the closet, but the red line is still over the blue one. In addition, we also consider the situations of $m$ and $\lambda$ at $\beta=$ 0.2 and $\alpha=0$, which show that the situation at $m=0.1$ is the same as $\beta=0.2$, and the $\lambda$ has very small effect on $I^{*}$ and $I^{1}$ when $\beta$ and $\alpha$ are too small. This also illustrates that the effect of $\beta$ and $\alpha$ is the biggest. The situations of other parameters are not given in here for their influences are very little. So, the equilibrium $E_{*}$ is locally asymptotically stable.

Furthermore, by observing the equations in system (5), we find that as $t \rightarrow+\infty, I(t)$ is monotone decreasing and $R(t)$ monotone increasing. The monotonicity of $S(t), W(t)$ is uncertain, but they are linear. From that, it is impossible to show limit cycle, from which the global stability of $E_{*}$ can be obtained.

We know the monotonicity of $S(t), W(t)$ is uncertain, depending of the parameter values, which have a certain meaning that will be interpreted later. At the end, the rumor will disappear by undergoing for a short time, and a portion of $S(0)=N^{*}$ becomes $R(\infty)$. Hence, the proportion of all spreader in the process of rumor spreading that had spread rumor to others or made statement about rumor in network should attract the attention of the public, which is called as the final scale of spreader, an important indicator of the severity of rumor spreading. Now, we give the expression for the final scale of spreader, denoted by $p(\infty)$.

Theorem 2. The final scale of spreader is

$$
\begin{gathered}
p(\infty)=\theta\left[1-e^{-\left(\beta / N^{*}+\lambda \alpha / k\right)} \int_{0}^{\infty} S(s) d s\right], \\
p(\infty)=\int_{0}^{\infty} 2 \xi_{1}\left[\frac{S(s)}{N^{*}}\right]^{2}+m \frac{S(s)}{N^{*}}+\xi_{2} \frac{S(s) R(s)}{N^{* 2}} d s .
\end{gathered}
$$

Proof. In the initial stages, $S(0) \ll I(0)$, so we assume $S(0)=$ $W(0)=0$ and $I(0) \approx N^{*}$, and at the end of rumor spreading, $S(\infty)=W(\infty)=0$. From the first equation in system (5), we have

$$
I(t)=N^{*} e^{-\int_{0}^{t}\left(\beta S(s) / N^{*}\right)+\alpha W(s) d s} .
$$

From the last equation in system (5), it can be obtained

$$
\int_{0}^{t} W(s) d s=\int_{0}^{t} \frac{\lambda}{k} S(s) d s-\frac{W(t)}{k} .
$$

Combining (10) and (11) and boundary conditions applied at 0 and $t \rightarrow \infty$, the following expression can be obtained:

$$
I(\infty)=N^{*} e^{-\int_{0}^{\infty}\left(\beta / N^{*}+\lambda \alpha / k\right) S(s) d s} .
$$




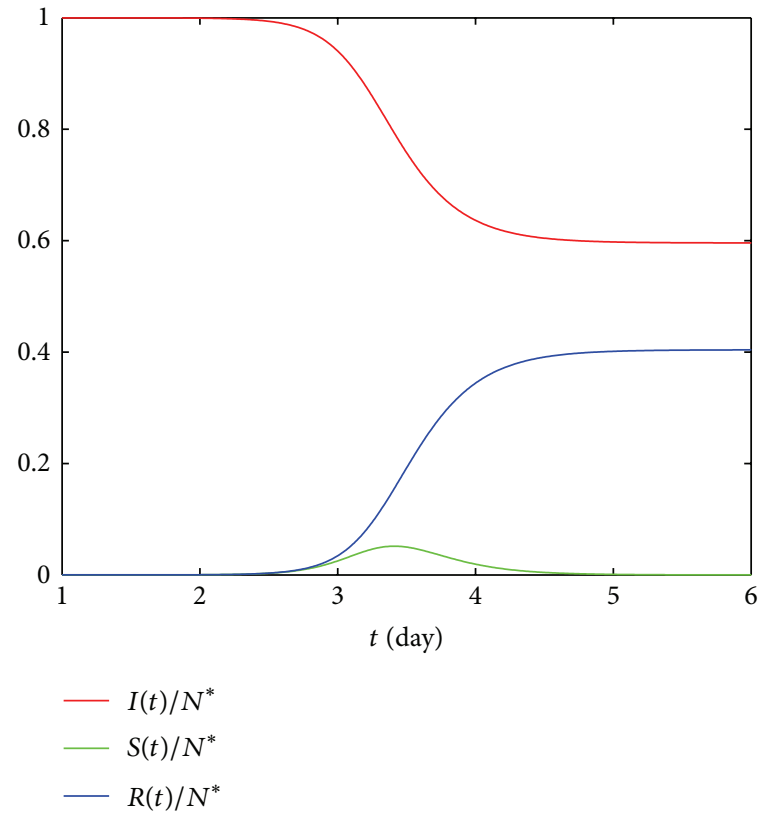

(a)

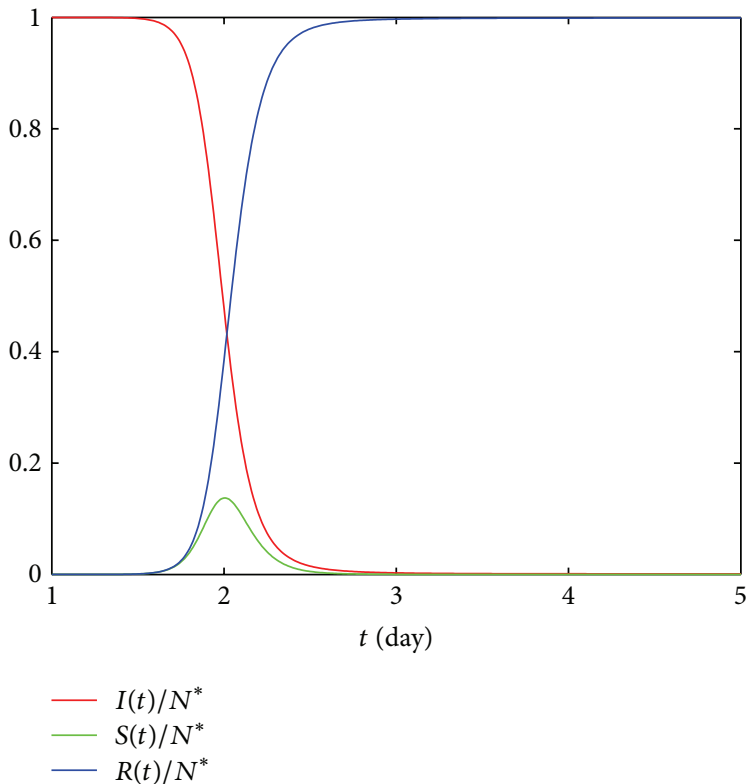

(b)

FIGURE 5: The number of each subclass in terms of time under different conditions, where $I(0)=5000000, S(0)=10, R(0)=0$, and $W(0)=0$. (a) $\beta=10, \alpha=0$; (b) $\beta=10, \alpha=0.00001$.

Substituting the relation $p(\infty)=\theta\left(N^{*}-I(\infty)\right) / N^{*}$, we can have the expression of the final spreader scale:

$$
p(\infty)=\theta\left[1-e^{-\left(\beta / N^{*}+\lambda \alpha / k\right) \int_{0}^{\infty} S(s) d s}\right]
$$

On the other hand, we can have directly another expression about the final scale from the last equation in system (5):

$$
p(\infty)=\int_{0}^{\infty} 2 \xi_{1}\left[\frac{S(s)}{N^{*}}\right]^{2}+m \frac{S(s)}{N^{*}}+\xi_{2} \frac{S(s) R(s)}{N^{* 2}} d s
$$

Due to the complexity of equations in model (5), we cannot give the expression only concluding $p(\infty)$. Comparing (8) with (9), Equation (8) is simpler and more convenient to use to assess parameters sensitivity.

\section{The Interpretation of Model Parameter Values}

In this section, we give the basis of parameter values in Table 1. Having no real data during the rumor spreading, we assume their values according to individual experience. Firstly, we assume that the average number of acquaintances one person meets per day is $C=30$ every day. When a spreader meets a susceptible, the probability that the spreader talks about the rumor and the susceptible is infected by the rumor is $\beta_{1}=2 / 3$ every day. After influenced by the rumor, half of the susceptible believe this rumor and begin to spread it, but the other half become the stiflers, not to spread this rumor. Thus, $\theta=0.5$. In other words, a third of people have no response, and a third of people begin to spread the rumor, and the rest, a third of people, become stiflers. So we get $\beta=\beta_{1} C=10$. When two spreaders contact, both of them discuss the rumor, and they lose interest in it for hearing it again and again. We assume the rate of losing interest is 0.5 every day, so $\xi_{1}=30 \times 0.5=15$. When a spreader contacts a stifler, the spreader will transmit the rumor, but the stifler tries to remove it. Assuming the probability that the spreader becomes a stifler is 0.5 , so $\xi_{1}=30 \times 0.5=15$. As time goes on, the spreader could be aware of the falsity content of the rumor by obtaining involved knowledge. As a result, the spreader also becomes a stifler at a rate of 0.1 . The most important and key point is disseminating of the rumor by the medium. Because the quantity of message in medium cannot be measured accurately, we can assume $1 \%$ of spreaders will disseminate the rumor into internet, and a person has 500 follower, then the rate that a spreader can disseminate the rumor through the medium is 5 . Moreover, due to the update of message on internet, the rumor will be hidden after 12 hours. So the submerged rate of message is 2 every day. With regard to the initial value adopted in this paper, we consider the total number of people is 5000010, where there are 10 spreaders, and $R(0)=W(0)=0$.

\section{Result and Sensitivity Analysis}

Taking no media message into account, we know that, when $d I(t) / d t \leq 0, I(t)$ will directly decrease to 0 , then the rumor cannot spread to others. When $d I(t) / d t>0, I(t)$ will first increase to its maximum at $t=T$, then decreases to $0 . d I / d t=$ 0 gives the threshold to justify whether the rumor spread to others can be obtained, which can be visually seen in Figure 3. 


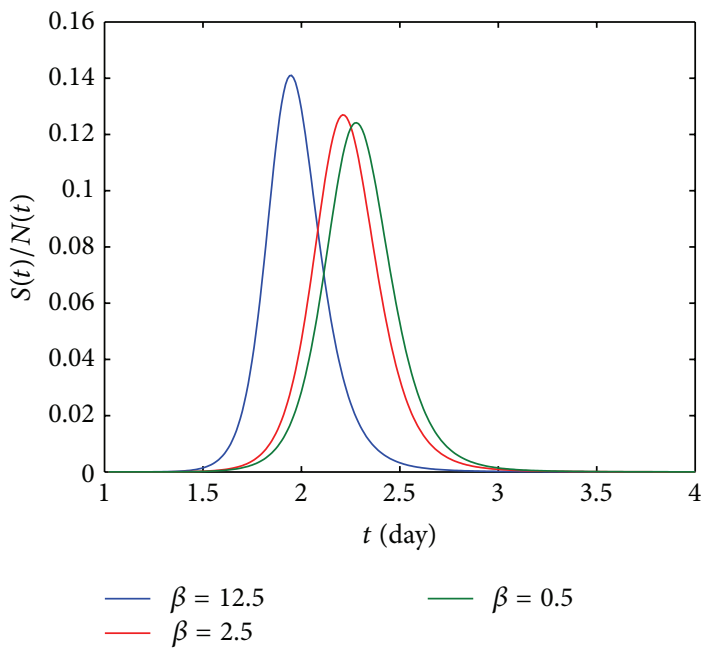

(a)

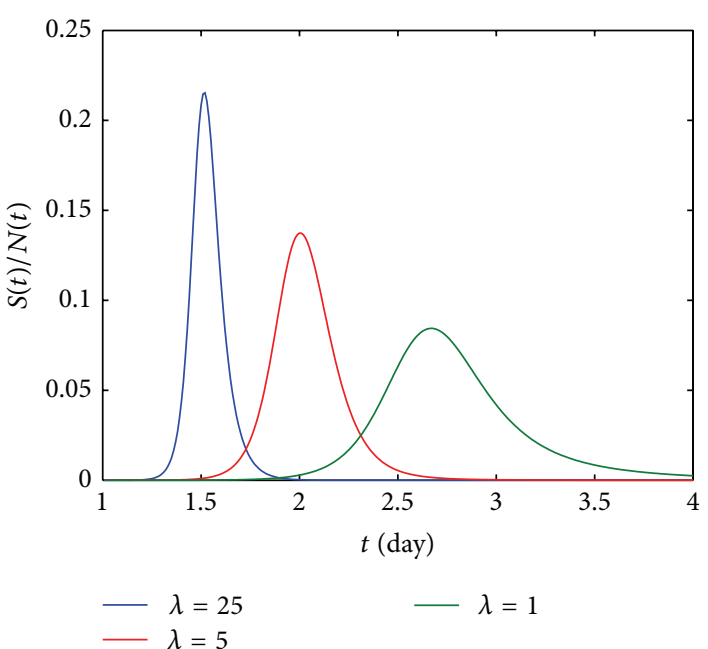

(c)

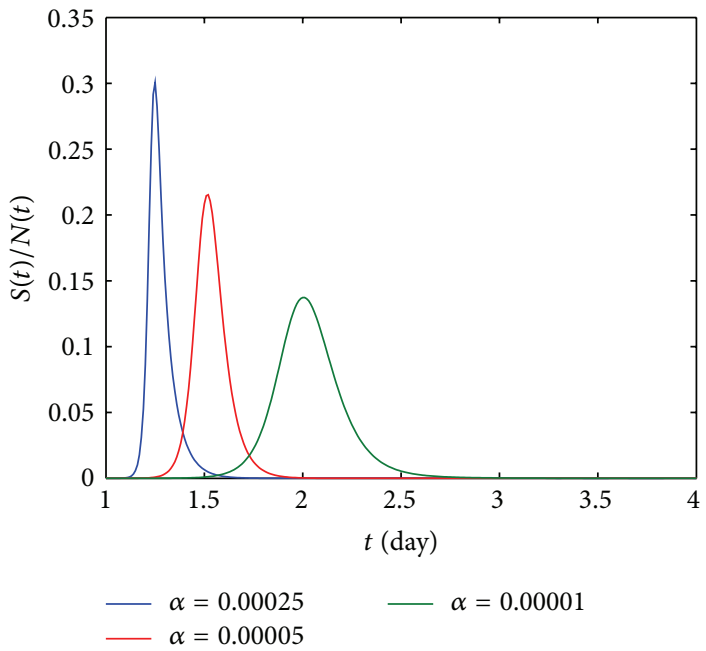

(b)

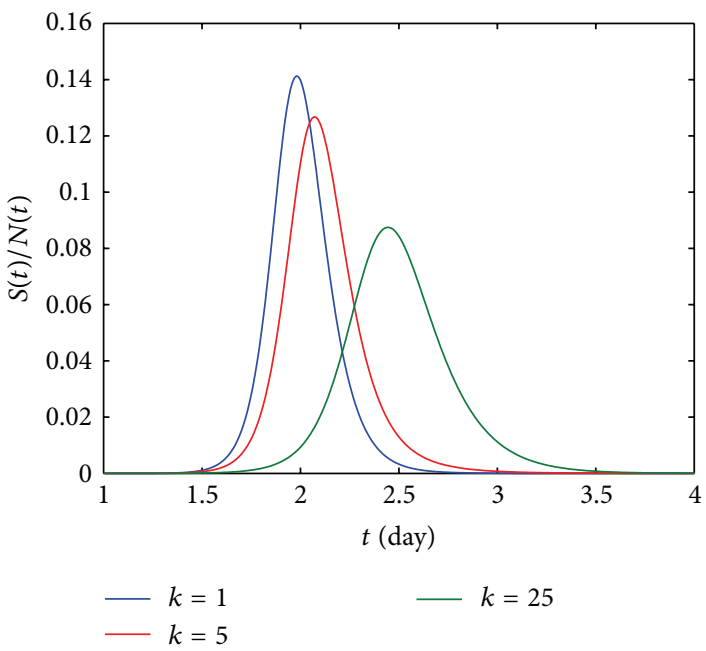

(d)

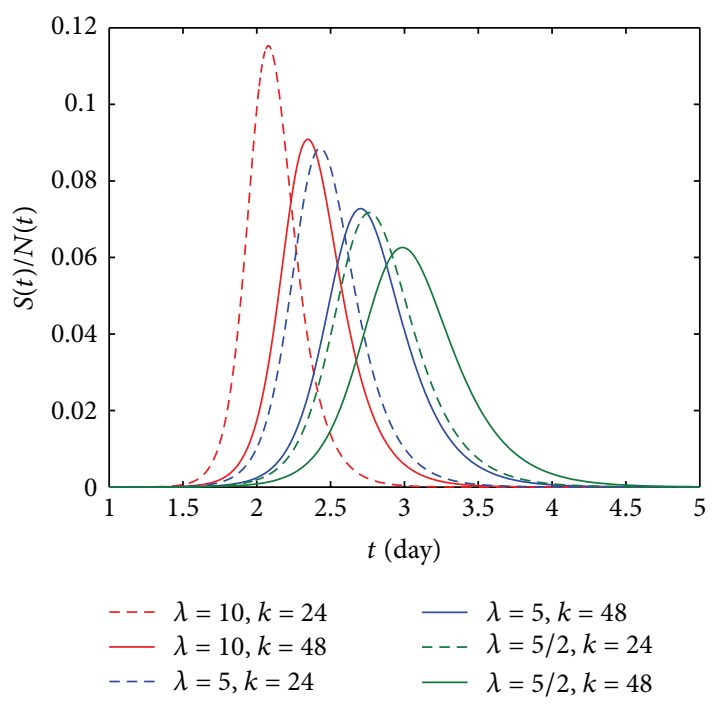

(e)

FIgURE 6: The proportion of spreader in terms of $\beta, \alpha, \lambda$, and $k$. 
However, taking the medium into consideration, the situation becomes more complex. $d I / d t \leq 0$ cannot give the threshold of rumor spreading, which needs to add the condition $d W / d t \leq 0$. For example, when $\beta=0.2$ and $\alpha=0.00001, d I / d t=-0.000602$. However, $d W / d t=50>0$, and the rumor can be disseminated out, seen in Figure 4. In fact, to control $d W / d t \leq 0$ is very difficult. In other words, in this case, it is more difficult to control the spreading of rumor. Moreover, because medium can enlarge the effect of rumor, the introducing of the medium leads to the big change of the solutions behavior, and even the transmission rate $\alpha$ is very small. We can draw the influence of medium on the rumor spreading in Figure 5. When $\alpha=0.00001$ that is very small, all people will know the rumor, and the peak of the proportion of spreader can arrive to about 0.15 from 0.05 . Moreover, observing the $x$-axis, the transmission speed becomes quicker, and the time of duration of rumor can be shortened.

What we are more interesting in is to find the key factors to affect the rumor diffusing. Firstly, we carry out sensitivity analysis of the spreader density about some parameters in Figure 6. From Figures 6(a)-6(b), we can compare the influence of $\beta$ with $\alpha$. When increasing 5 times to parameters $\alpha$, the change of $S(t) / N(t)$ will arrive 1.5 times, but for $\beta$ it has very little change. From that, we can know that the role of the medium is more important during the transmission of rumor. Moreover, Figures 6(c)-6(d) have shown that reducing $\lambda$ or increasing $k$ can reduce the peak of the proportion of spreader and delay the arrival time of the peak. However, $\lambda$ has larger influence than $k$ on the fraction of spreader. That means the prevention of rumor releasing is more important than the eliminating of existing rumor. So, the enhancing managing network is a vital measure. To look at the influence of $\lambda$ and $k$, we give a detailed discussion in Figure 6(e). With the decreasing of $\lambda$, the reduction rate is decreasing, and the effect of $k$ becomes smaller; that is, when $\lambda$ is very small, the sensitivity and effect of $\lambda$ becomes smaller. In turn, when the $k$ becomes bigger, the influence of $\lambda$ also becomes smaller. Therefore, reducing $\lambda$ or increasing $k$ is good measure to control rumor spreading, but the effects are not always obvious, which need detailed real data to confirm. Besides, it is remarkable that changing the $\alpha, \beta, \lambda$, and $k$ can reduce the peak, but the width of cure becomes bigger. So, there are more questions that need in-depth discussions in future work.

\section{Discussion}

With the new pattern of rumor spreading corresponding to the appearance of internet, we establish an ISRW dynamical model considering the medium as a subclass. Beside the dynamical analysis of the model, that is, the stability of the equilibrium and final spreader scale, we mainly explore the mechanism of spreading between individuals and from medium-to-individual. By numerical simulation, we find that, due to the introduction of the medium, the mechanism becomes complex. Controlling the rumor spreading need to not only control the derivative of the spreader subclass, but also control change rate of the information about rumor in medium. However, it is difficult to control the information about rumor in medium. By comparing the transmission rate $\beta$ between individuals with the transmission rate $\alpha$ of medium-to-individuals, we find the effect of $\alpha$ is larger than $\beta$ for $\alpha$ can enlarge the effect of rumor, seen in Figure 6 . From Figure 5, we also can see when $\alpha$ is very small, its influence is bigger relatively. It is thus clear that consideration of medium during the rumor spreading is necessary. Besides, in Figures $6(\mathrm{c})$ and $6(\mathrm{~d})$, we find $\lambda$ has a larger influence than $k$. That is to say, controlling the effusion of rumor is more important than deleting existing rumor. Thus, on the one hand, government should enhance the management of internet, such as the establishing of real-name registration system. On the other hand, relevant legal institutions for the rumor creator and spreader on internet that can be tracked in network should be established. If obtaining all real data about rumor spreading, we can apply the model to quantify various measures, which is very helpful. Resorting to the methods proposed in this paper, the authorities can propose efficient measures to control the rumor spreading to keep the stabilization of society and development of economy.

The model we consider in this paper is deterministic, which means it is a fixed system. Once the same initial conditions and the same parameter values are given, the same trajectory is always obtained. Obviously, there exists a certain gap with the real situation. Clearly, the result is just a situation which appears with a certain probability. So some papers adopted the stochastic model to investigate the transmission process [21-24]. During the rumor spreading, the behavior of individuals may have a certain randomness, that is, the number of contact with others and medium every day and the frequency of being on the net are not constant. Therefore, that the parameters $\beta, \alpha, \xi_{1}, \xi_{2}$, and $\lambda$ are added with a noise (randomness) is more rational. In this case, the steady state is not fixed and is an oscillating curve. So the deterministic model is a mean situation, and the stochastic model is more detailed. The more detailed discussion will be our future work.

Besides, our model is built based on mean and rough conditions. In fact, during rumor spreading there are more human behavior, such as forget-remember mechanism [16] which is not considered in our model. Moreover, the contact number and followers of every people should be different, but it is assumed to be constant in this paper. In emergency situation, authority plays a role of manager [5] that usually publishs some statement to clarify rumors. In this paper, we do not consider it in the way that can confirm rumor and protect people from the rumor infection. These are the directions of research in our future work. In a word, this paper is just an initial working, and lots of work need more prefect models to study.

\section{References}

[1] K. Kawachi, M. Seki, H. Yoshida, Y. Otake, K. Warashina, and H. Ueda, "A rumor transmission model with various contact interactions," Journal of Theoretical Biology, vol. 253, no. 1, pp. 55-60, 2008. 
[2] J. Kostka, Y. A. Oswald, and R. Wattenhofer, "Word of mouth: rumor dissemination in social networks," in Structural Information and Communication Complexity, vol. 5058 of Lecture Notes in Computer Science, pp. 185-196, Springer, Berlin, Germany, 2008.

[3] Z. L. Zhang and Z. Q. Zhang, "An interplay modal for rumour spreadingand emergency development," Physica A, vol. 388, no. 19, pp. 4159-4166, 2009.

[4] M. Kosfeld, "Rumours and markets," Journal of Mathematical Economics, vol. 41, no. 6, pp. 646-664, 2005.

[5] L. J. Zhao, Q. Wang, J. J. Cheng et al., "The impact of authorities' media and rumor dissemination on theevolution of emergency," Physica A, vol. 391, no. 15, pp. 3978-3987, 2012.

[6] L. J. Zhao, J. J. Wang, Y. C. Chen et al., "SIHR rumor spreading model in socialnetworks," Physica A, vol. 391, no. 7, pp. 24442453, 2012.

[7] A. Sudbury, "The proportion of the population never hearing a rumour," Journal of Applied Probability, vol. 22, no. 2, pp. 443446, 1985.

[8] K. Kawachi, "Deterministic models for rumor transmission," Nonlinear Analysis: Real World Applications, vol. 9, no. 5, pp. 1989-2028, 2008.

[9] A. Rapoport, "Spread of information through a population with socio-structural bias. I. Assumption of transitivity," Bulletin of Mathematical Biophysics, vol. 15, pp. 523-533, 1953.

[10] A. Rapoport, "Spread of information through a population with socio-structural bias. II. Various models with partial transitivity," Bulletin of Mathematical Biophysics, vol. 15, pp. 535546, 1953.

[11] A. Rapoport and L. I. Rebhun, "On the mathematical theory of rumor spread," Bulletin of Mathematical Biophysics, vol. 14, pp. 375-383, 1952.

[12] D. J. Daley and D. G. Kendall, "Epidemics and rumours," Nature, vol. 204, article 1118, 1964.

[13] D. P. Maki and M. Thompson, Mathematical Models and Applications, Prentice-Hall, Englewood Cliffs, NJ, USA, 1973.

[14] B. Pittel, "On a Daley-Kendall model of random rumours," Journal of Applied Probability, vol. 27, no. 1, pp. 14-27, 1990.

[15] C. Lefèvre and P. Picard, "Distribution of the final extent of a rumour process," Journal of Applied Probability, vol. 31, no. 1, pp. 244-249, 1994.

[16] J. Gu, W. Li, and X. Cai, "The effect of the forget-remember mechanism onspreading," European Physical Journal B, vol. 62, no. 2, pp. 247-255, 2008.

[17] L. A. Huo, P. Q. Huang, and X. Fang, "An interplay model for authorities' actions and rumor spreading in emergency event," Physica A, vol. 390, no. 20, pp. 3267-3274, 2011.

[18] L. J. Zhao, Q. Wang, J. J. Cheng, Y. C. Chen, J. J. Wang, and W. Huang, "Rumor spreading model with consideration of forgetting mechanism: a case of online blogging live journal," Physica A, vol. 390, no. 13, pp. 2619-2625, 2011.

[19] K. L. Cooke, "Stability analysis for a vector disease model," The Rocky Mountain Journal of Mathematics, vol. 9, no. 1, pp. 31-42, 1979.

[20] Y. Wang, Z. Jin, Z. M. Yang, Z. K. Zhang, T. Zhou, and G. Q. Sun, "Global analysis of an SIS model with an infective vector on complex networks," Nonlinear Analysis: Real World Applications, vol. 13, no. 2, pp. 543-557, 2012.

[21] G. Q. Sun, L. Li, Z. Jin, and B. L. Li, "Effect of noise on the pattern formation in an epidemic model," Numerical Methods for Partial Differential Equations, vol. 26, no. 5, pp. 1168-1179, 2010.
[22] M. Krstić, "The effect of stochastic perturbation on a nonlinear delay malaria epidemic model," Mathematics and Computers in Simulation, vol. 82, no. 4, pp. 558-569, 2011.

[23] C. Y. Ji, D. Q. Jiang, Q. S. Yang, and N. Z. Shi, "Dynamics of a multigroup SIR epidemic model with stochastic perturbation," Automatica, vol. 48, no. 1, pp. 121-131, 2012.

[24] V. Mendez, D. Campos, and W. Horsthemke, "Stochastic fluctuationsof the transmission rate in the susceptible-infectedsusceptible epidemicmodel," Physical Review E, vol. 86, no. 1, Article ID 011919, 8 pages, 2012. 


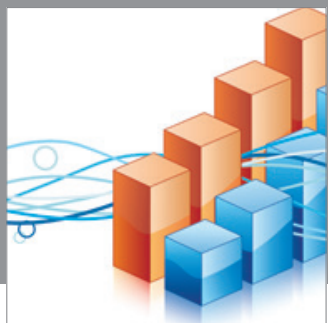

Advances in

Operations Research

mansans

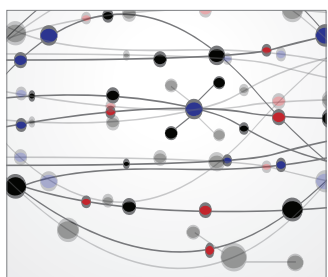

The Scientific World Journal
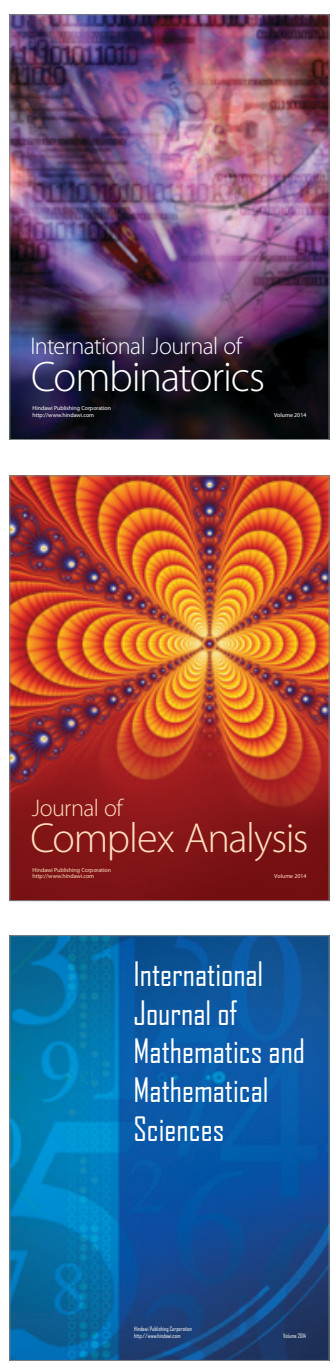
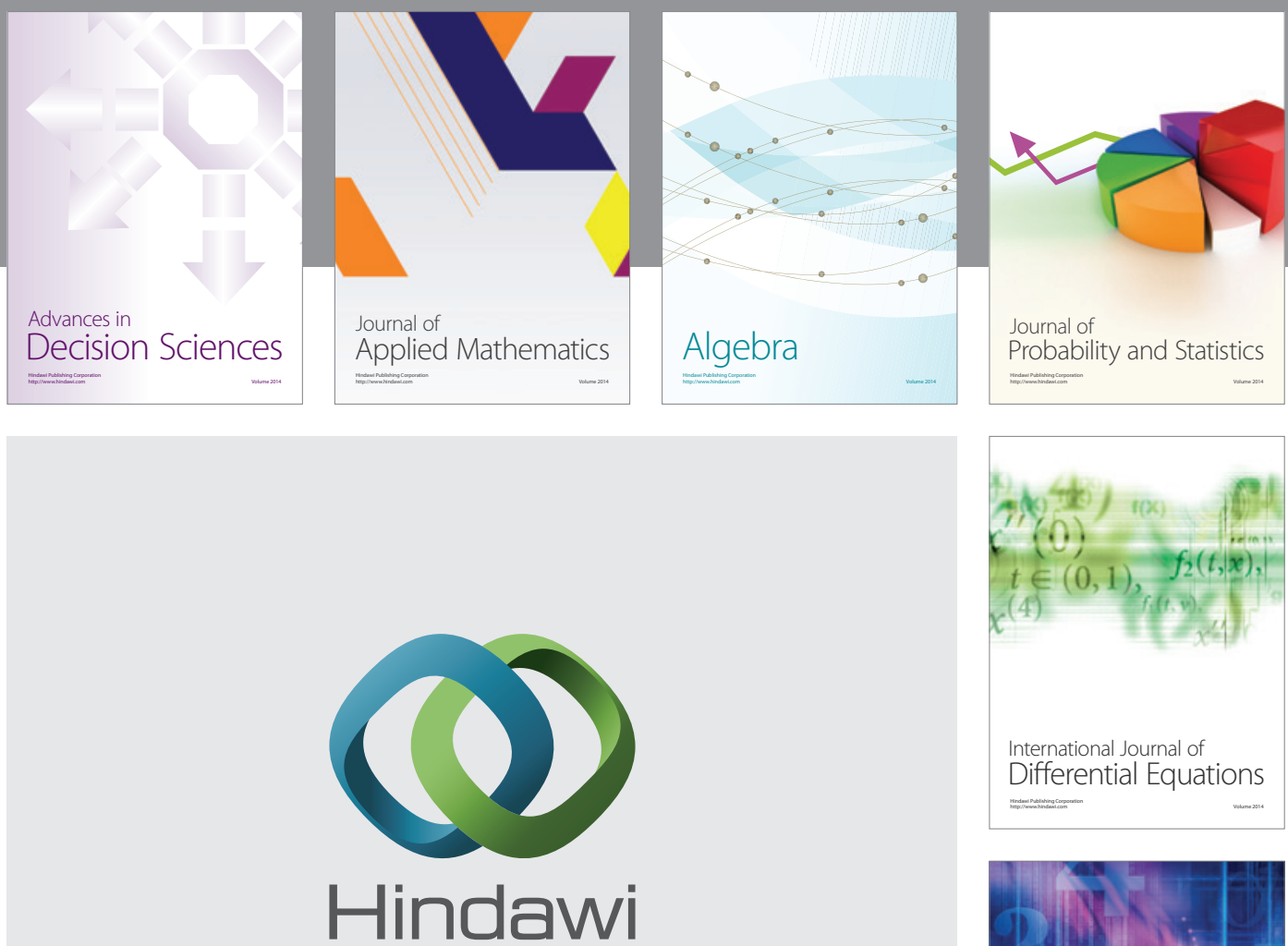

Submit your manuscripts at http://www.hindawi.com
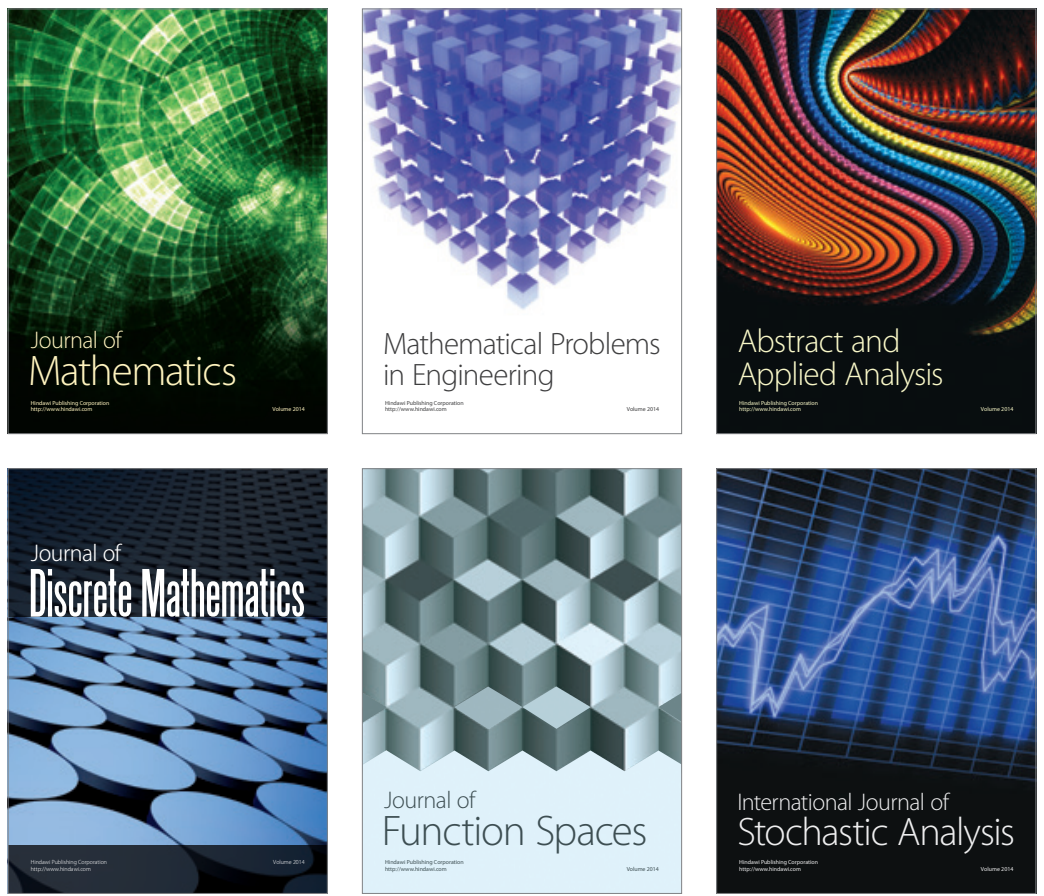

Journal of

Function Spaces

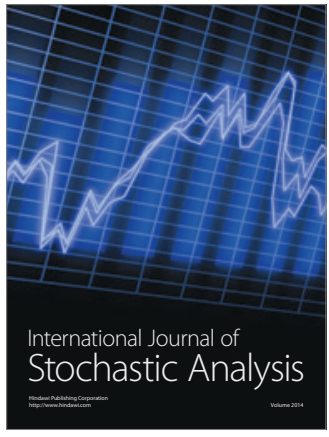

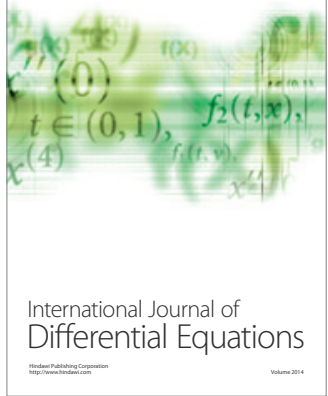
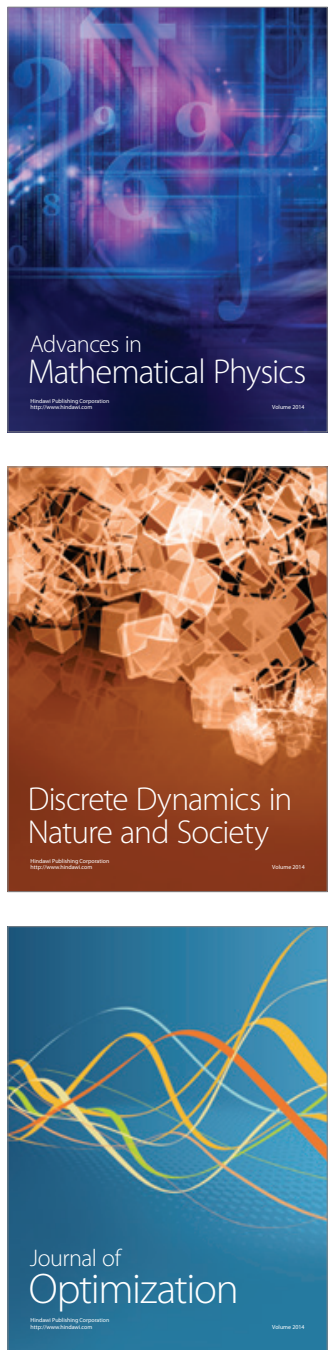